\title{
European Physical Society
}

Postal Address:

POB 69

CH-1213 Petit-Lancy 2

T. (22) $931130 / 31 / 32$

Tx. 428024 eps ch

Cables:Europhysics Genève
Location:

27. Chemin de la Vendée

Petit-Lancy, Geneva

Access:

Chemin du Banc-Bénit
Supplementary Secretariat:

Fö u. 68, 1st floor / No. 141

H-1027 Budapest Executive Secretary:

T. (1) 358386
G. Thomas

\section{Past Presidents}

1968-1970:

G. Bernardini, Pisa

1970-1972

E. Rudberg, Stockholm

1972-1976:

H.B.G. Casimir, Eindhoven 1976-1978:

I. Ursu, Bucharest

1978-1980:
A. Zichichi, Bologna
1980-1982:
A.R. Mackintosh, Copenhagen
1982-1984:
J. Friedel, Orsay
1984-
G.H. Stafford, Oxford

G.H. Stafford, Oxford

\section{Honorary Members}

H.O.G. Alfven, Stockholm, Sweden

E. Amaldi, Rome, Italy

G. Bernardini, Pisa, Italy

N.N. Bogolubov, Moscow, USSR

H.B.G. Casimir, Heeze, The Netherlands

F. Hund, Göttingen, Red. Rep. of Germany

Sir Nevill Mott, Milton Keynes, UK

L.E.F. Néel, Meudon, France

V.F. Weisskopf, Cambridge, USA

\section{Individual Ordinary Members}

In June 1985 the number of Individual Ordinary Members on the books of the Society totalled 3803 . The names of the nine delegates to Council who represent these members are given below.

\section{I.O.M. Delegates in Council}

Delegates are elected by a ballot of all the Individual Ordinary Members for a term of three years. The term is automatically extended if the delegate is elected to the Executive Committee.

+ J. Ernest, Marcoussis

B. Feuerbacher, Cologne

+ W. Heine, Cambridge

+ F. Janouch, Stockholm

F. Mezei, Budapest

E. Skrzypczak, Warsaw

W. Thirring, Vienna

M. Tosi, Trieste

A. Wapstra, Amsterdam

+ in last year of term

\section{Executive Committee}

President: G.H. Stafford

St. Cross College

Oxford OX1 3LZ, UK

T. (865) 512411

Vice-President: W. Buckel

Physikalisches Institut der Universität

Engesserstrasse 7

E-7500 Karlsruhe

T. (721) 6083451

Secretary: W.J. Merz

Laboratories RCA Ltd.

Badenerstrasse 569

$\mathrm{CH}-8048$ Zürich

T. (1) 4926350

Vice-Secretary: N. Kroo

Central Research Institute for Physics

POB 49

$\mathrm{H}-1525$ Budapest

T. (1) 696575

Treasurer: D. Kuhn

Institut für Experimentalphysik

Abteilung Hochenergiephysik

Sillgasse 8

A-6020 Innsbruck

T. (5222) 724 ext. 6670

Vice-Treasurer: E.W.A. Lingeman Institute for Nuclear Physics Research POB 4395

NL-1009 Amsterdam

T. (20) 592911 ext. 2013/1012
Members:

B. Dreyfus

CEN - Département de Recherche Fondamentale

$85 \mathrm{X}$

F-38047 Grenoble Cedex

T. (76) 974111 ext. 5024

J.M. Gilles

Département de Physique

Facultés Universitaires N.-D. de la Paix

Rue de Bruxelles 61

B-5000 Namur

T. (81) 229061 ext. 2897

O.V. Lounasmaa

Low Temperature Laboratory

Helsinki University of Technology

SF-Espoo 15

T. (80) 4512453

K.K. Rebane

Academy of Sciences of the Estonian SSR

Kohtu 6

USSR-200103 Tallinn

T. 442129

\section{R.A. Ricci}

Laboratori Nazionali di Legnaro

Via Romea, n. 4

1-35020 Legnaro (Padova)

T. (49) 641200

\section{- Ampere Group (220) 1}

\section{Del. G.J. Béné}

Pres. K.H. Hausser

Max-Planck Institut für Medizinische

Forschung, Jahnstrasse 29

D-6900 Heidelberg

Sec-Gen. G.J. Béné

Université de Genève, Section de

Physique, quai Ernest-Ansermet 24

CH-1211 Geneva 4

T. (22) 219355

Austrian Physical Society (806) 2

Dels. G. Bauer

$$
\text { D. Kuhn }
$$

Pres. H. List

AVL-LIST GmbH

Kleiststrasse 48

A-8011 Graz

Sec. R. Dobrozemsky

Physik-Institut

Osterreichisches Forschungszentrum

A-2444 Seibersdor

T. $(2254) 802270$

\section{Member Organizations}

(X) $Y$

$X=$ No. of members in the Society

or Group

$Y=$ No. of delegates to Council

Bulgarian Acad. of Sciences (105) 1

Del. P. Simova

Pres. A. Balevski

Sec-Gen. G. Bliznakov

Foreign Relations Department

7 November str. No. 1

Sofia BG

Del. J.M. Gilles

Pres. J.M. Gilles

Facultés Universitaires Notre-Dame

de la Paix

Rue de Bruxelles 61

B-5000 Namur

Gen-Sec. P. Fettweis

CEN/SCK

Boeretang 200

B- $2400 \mathrm{Mo}$

T. (14) 311801
T. 877731

Danish Physical Society (357) 1

Del. P.G. Hansen

Pres. H. Smith

Sec. J. Jensen

H.C. Orsted Institute

Universitetsparken 5

DK-2100 Københaven 0

T. (1) 353133
Auditors:

G. Landwehr, Würzburg

P. Radvanyi, Gif-sur-Yvette

FIDES， Geneva
Dept. of Gen. Physics and Astro nomy, USSR Acad. of Sciences (2000) 4

Dels, J. Pozhela

$$
\text { K.K. Rebane }
$$

Pres. A.M. Prokhorov

Sci-Sec. S. Markyanov

USSR Academy of Sciences

Leninskyi Prospekt 14

USSR-Moscow B-71

T. 2322910

Eötvös Lorand Physical Society (625) 2

Dels. I. Abonyi N. Kroo

Pres. G.J. Csikai

Institute of Experimental Physics Kossuth University, Bem tér 18/a POB 105

H-4001 Debrecen

Sec-Gen. N. Kroo

Eötvös Lorand Fisikai Tarsulat

Anker köz 1, POB 240

$\mathrm{H}-1368$ Budapest

T. (1) 227040 
Finnish Physical Society (772) 2

Dels. R. Hämäläinen

O.V. Lounasma

Pres. H.I. Miettinen

Research Inst. for Theoretical Physics

University of Helsinki

Siltavuorenpenger 20 D

SF-00170 Helsinki 17

Sec. M. Nordberg

Department of Physics

University of Helsinki

Siltavuorenpenger 200

SF-00170 Helsinki 17

T. (0) 650211

French Physical Society (2700) 4

Dels. B. Dreyfus

M. Jacob

P. Radvany

M. Veyssie

Pres. M. Jacob

CERN

TH Division

$\mathrm{CH}-1211$ Geneva 23

Sec-Gen. M. Veyssie

Société Française de Physique

33 rue Croulebarbe

F-75013 Paris

T. (1) 7073298

German Physical Society (8450) 5

Dels. W. Buckel

O.G. Folberth
G. Grieger
G. Landwehr
J. Trümper

G. Landwehr

Pres. J. Treusch

Institut für Physik der Universität

Postfach 500500

D-4600 Dortmund 50

Gen-Sec. R. Poerschke

Deutsche Physikalische Gesellschaft

Hauptstrasse 5

D-5340 Bad Honnef

T. (2224) 71061

Hellenic Physical Society (55)

Pres. N.G. Antoniou

Hellenic Physical Society

6 Griveon Street

GR-Athens 144

T. (1) 635701

Icelandic Physical Society (45) 1

Pres. T. Jonsson

Science Institute, University of Iceland

Dunhaga 3

IS-107Reykjavik

Sec. S. Sigurdisson

Karsnesbraut 18

IS-200 Kopavogi

The Institute of Physics (12000) 5

Dels. Sir Robert Clayton

L. Cohen

D.H. Martin

Sir Alec Merrison

G.H. Stafford

B.L.H. Wilson

Pres. Sir Robert Clayton

The General Electric Co. Ltd.

Hirst Research Centre, East Lane

Wembley, Middlesex HA9 7PP, UK

Exec-Sec. L. Cohen

The Institute of Physics

47 Belgrave Square

London SW1X 8QX, UK

T. (1) 2356111 Tx. 918453

Institute Ruder Boskovic (100) 1

Del. S. Popovic

Dir-Gen. S. Kveder

Sec. N. Zovko

Institute Ruder Boskovic

Bijenicka 54, POB 1016

YU-41001 Zagreb

T. (41) 435111 Tx. 21383

Israel Physical Society (310) 1

Del. D. Horn

Pres. D. Horn

Sec. A. Gotsman

Department of Physics \& Astronomy

Tel-Aviv University

Tel-Aviv, Israel

T. (3) 420587
Italian Physical Society (1100) 3

Dels. I. Ortalli

$$
\begin{aligned}
& \text { R.A. Ricci } \\
& \text { A. Rubbino }
\end{aligned}
$$

Pres. R.A. Ricci

Laboratori Nazionali INFN

Via Romea 4

1.35020 Legnaro (Padova)

Sec. E. Mazzi

Società Italiana di Fisica

Via L. degli Andalò 2

1-40124 Bologna

T. (51) 331554

The Netherlands' Physical Society

(2966) 4

Dels. F.R. de Boer

E.W.A. Lingeman

J.K. van Deen

F. van der Woude

Pres. F. van der Woude

Lab. voor Vaste Stoffysica

Melkweg 1

NL-9718 EP Groningen

Sec. F.R. de Boer

Nederlandse Natuurkunde Vereniging

Princetonplein 5, POB 80000

NL-3508 TA Utrecht

T. (30) 532329

Norwegian Physical Society (627)

Dels. T. Engeland

$$
\text { R.S. Sigmond }
$$

Pres. E. Osnes

Department of Physics

University of Oslo

$\mathrm{N}$-Blindern, Oslo 3

Sec. G. Jarrett

Institutt for Atomenergi, РОB 40

$\mathrm{N}-2007 \mathrm{Kjeller}$

T. (2) $712560 \quad$ Tx. 16361 atom n

Phys. Sect. Union of Czechoslovak

Mathematicians and Physicists

(500) 2

Dels. P. Povinec

S. Safrata

Pres. S. Krupicka

Sec. I. Veltrusky

Czechoslovak Academy of Sciences

$\mathrm{Na}$ Slovance 2

CS-18040 Prague 8

T. (422) $842241 / 823603$

Tx. 122018 atom c

Physical Society of the German

Democratic Republic (1260) 3

Dels. D. Hommel

U. Krecker

K. Thiessen

Pres. R. Rompe

Sci-Sec. R. Linke

Physikalische Gesellschaft der

Deutschen Demokratischen Republik

Am Kupfergraben 7

DDR-1080 Berlin

T. (2) 2000691

Physical Sect. Union of Yugoslav

Societies of Mathematicians

Physicists and Astronomers (200) 1

Del. D. Jovic

Pres. V. Dajovic

Gospodar Jevremova 45

YU-11000 Belgrade

Sec-Gen. P. Obradovic

Institute of Mathematics and Physics

University of Titograd

Vase Raickovica 25

YU-81000 Titograd

T. (81) 52224

Polish Physical Society (1890) 4

Dels. J. Konopka

$$
\begin{aligned}
& \text { K. Rosinski } \\
& \text { T. Skalinski }
\end{aligned}
$$

s. T. Skalinski

Institute of Physics of the

Polish Academy of Sciences

Al. Lotnikow $32 / 46$

PL-02668 Warsaw

Gen-Sec. J. Konopka

Polskie Towarzystwo Fizyczne

Hoza 69

PL-00681 Warsaw

T. (22) 212668
Portuguese Physical Society (200) 1

Del. F. Duarte Santos

Pres. J.M. Araujo

Department of Physics

Faculty of Sciences

P-4000 Porto

T. (2) 310290 Tx. 28109 INESCN

Sec. F. Duarte Santos

Sec Ext Rel. J. Bessa Sousa

Sociedade Portuguesa de Fisica

Av. da Republica 37, $4^{\circ}$

\begin{tabular}{|c|}
\hline $\begin{array}{l}\text { for Physics (300) } 1 \\
\text { Del. A. Corciovei } \\
\text { Pres. I. Ursu } \\
\text { National Council for Science } \\
\text { and Technology } \\
\text { Roma Street } \\
\text { Bucharest R }\end{array}$ \\
\hline $\begin{array}{l}\text { Sec. A. Corciovei } \\
\text { Central Institute of Physics } \\
\text { POB MG6 } \\
\text { Bucharest R } \\
\text { T. } 807040 \text { ext. } 3115 \\
\text { Tx. } 11397 \text { fiatom r }\end{array}$ \\
\hline
\end{tabular}

P-1000 Lisbon

T. (1) 773251

Royal Irish Academy (60) 1

Del. A. Thompson

Pres. E.F. Fahy

Sec. A. Thompson

Irish National Committee for Physics

19 Dawson Street

Dublin 2 IRL

T. (31) 762570

Royal Spanish Society of Physics

(200) 1

Del. J. Campos

Pres. C. Sanchez del Rio

Gen-Sec. M.R. Heras Celemin

Real Sociedad Española de Fisica

Facultades de Ciencias

Ciudad Universitaria

E-Madrid 3

T. (1) 2433879

Swedish Physical Society (1036) 2

Dels. R. Hellborg

B.E.Y. Svensson

Pres. B.E.Y. Svensson

Institute of Theoretical Physics

University of Lund

Sölvegatan 14

S-22362 Lund

Sec. R. Hellborg

Institute of Physics

University of Lund

Sölvegatan 14

S-22362 Lund

T. (46) 107644

Swiss Physical Society (1160) 3

Dels. J.P. Blaser

$$
\begin{aligned}
& \text { S. Steinemann } \\
& \text { W.J. Merz }
\end{aligned}
$$

W.J. Merz

Sec. P. Grieder

Inst. f. Theoretische Physik

Universität Bern

Sidlerstrasse 5

$\mathrm{CH}-3012$ Bern

T. (31) 658631

Turkish Physical Society (302)

Del. M. Serdaroglu

Pres. E. Inönü

Tanova Sitesi No, 3

Bebek

Istanbul TR

Gen-Sec. M. Serdaroglu

Bogaziçi Universitesi Fizik Bölümü

P.K. 2, Bebek

Istanbul TR

T. (1) 1631500 ext. $604 / 630$

Tx. Boun tr 26411
Collaborating Societies

American Physical Society

335 East 45th St.

New York

N.Y, 10017 USA

T. (212) 6827341

Société Francaise des Spécialistes

d'Astronomie (SFSA)

Pres. J. Delhaye

Observatoire de Paris

61 Avenue de l'Observatoire

F-75014 Paris

Società Astronomica Italiana

Vice-Pres. M. Fulchignoni

28 Via Brera

1-20121 Milano

Nederlandse Astronomenclub

Sec. T.A.Th. Spoelstra

Radiosterrenwacht

Postbus 2

NL-7990 AA D wingeloo

Société Française d'Optique

Ch. J. Bulabois

Faculté des Sciences et Techniques

Université de Franche-Comté

F-25030 Besançon

Société Suisse d'Optique et de Microscopie Electronique (SSOME) Ch. E. Mathieu

Wild Heerbrugg AG

$\mathrm{CH}-9435$ Heerbrugg 


\section{Applied Physics and Physics in Industry}

Chair. G. Pfister

Cerberus AG

$\mathrm{CH}-8708$ Männedor

T. (1) 9226263

F. Aussenegg, Karl-Franzens-University, Graz

H. de Kluiver, FOM, Nieuwegen

J. Ernest, C.IT, Alcatel, Paris

E. Feldtkeller, Siemens AG, Munich

J. Gyulai, Central Research

Institute for Physics, Budapest

J. Halfdanarson, Akranes

D. Lundqvist, Bromma

A. Olès, Academy of Mining and

Metallurgy, Cracow

C.P. O'Toole, Royal Irish Acad., Dublin

A. Paoletti, CNR, Rome

S. Popovic, Institute of Physics,

Belgrade

A. Prizam, Weizmann Inst. of Science,

Rehovot

G. Raoult, University, Clermont-Ferrand

H. Reiner, SEL, Stuttgart

F.C. Rodrigues, Nat. Lab. for Eng. and Ind. Technology, Sacavem

F. Scarlat, Central Institute of Physics, Bucharest

Z. Sigut, Central Research Inst., Pilsen

A. Sokollu, Marmara Research

Institute, Istanbul

K.A. Valiev, Inst. of General Physics, Moscow

B.L.H. Wilson, Plessey Ltd., Towcester

R. Zelazny, JET, Abingdon

\section{Physics Education}

Chair. J. Depireux

Institute of Physics

University of Liege

Sart Tilman

Sart Tilman

T. (41) 569811

Sec. M.G. Ebison

Education Office

The Institute of Physics

47 Belgrave Square

London SW1X 80X, UK

T. (1) 2356111

I. Abonyi, University, Budapest

E. Adlerova, Slovak Technical

University, Bratislava

E.R. Andrew, University, Nottingham

J. Auth, DDR National Committee

for Physics, Berlin

D.A. Blackburn, Open Univ., Oxford

G. Bonera, Department of Theoretical and Nuclear Physics, Pavia

G. Born, Gesamthochschule, Duisburg

T.P. Burke, University, Dublin

J. Campos, University, Madrid

A. Gotsman, University, Tel-Aviv

A. Janner, University, Nijmegen

N. Joel, UNESCO, Paris

F. Kaczmarek, Adam Mickiewicz Univ.

Poznan

K.J. Knutsen, Trondheim

J. Konopka, University, Warsaw

S. Kozel, Technical Inst., Moscow

C. Leubner, University, Innsbruck

M. Martinis, Ruder Boskovic Inst., Zagreb

A. Meessen, Solid State Physics Unit, Louvain-la-Neuve

V. Meisalo, University, Helsinki

R. Nasuhoglu, Faculty of Science,

Ankara

C. Plavitu, Central Institute of Physics, Bucharest

A. Raev, Faculty of Physics, Sofia

S. Raither, UNESCO, Paris

L. Silverberg, University, Lund

S. Steinemann, University, Lausanne

M. Taieb, Palais de la Découverte, Paris

P. Theodorsson, Univ, of Iceland, Reykjavik

M.F. Thomaz, University, Aveiro

P. Thomsen, Royal School of

Educational Studies, Copenhagen

Chair. J.M. Araujo

Laboratorio de Fisica

Universidade do Porto

P.4000 Porto

T. (2) 310290 ext. 230

ir. S. Methfesse

Sec. R. Hahn

Institut für Experimentalphysik

Ruhr Universität

Postfach 102148

D-4630 Bochum

T. (234) $7003649 / 50$

O. Akyuz, Bogaziçi Univ., Istanbul

R. Behrisch, MPI for Plasma Physics,

Garching

B. Cagnac, Univ. Pierre et Marie Curie, Paris

M.J. Colles, Heriot-Watt Univ. Edinburgh

I.A. Dorobantu, Central Institute of Physics, Bucharest

J. Fousek, Institute of Physics, Prague

A. Gotsman, University, Tel-Aviv

J. Heijn, NNN Physics Laboratory, Utrecht

I. Iori, Institute of Physics, Milan

A. Kujawski, Polish Academy of

Sciences, Warsaw

A. Lösche, University, Leipzig

Lovas, Central Research Institute for

Physics, Budapest

N. Lund, Danish Space Research Inst., Lyngby

D. Lundqvist, Bromma

S. Magnusson, Univ. of Iceland,

Reykjavik

J.B. Meneses e Sousa, University, Porto

W.J. Merz, RCA Laboratories, Zürich

M. Nardelli, University, Parma

W.S. Newman, Geneva

J.S. Nilsson, Institute of Theoretical Physics, Göteborg

R. Picchi, CERN, Geneva

J. Smidt, Laboratory for Technical

Physics, Delft

S.D. Smith, Heriot-Watt University, Edinburgh

J.-P. Swings, Institute of Astrophysics, Cointe-Liège

G. Thomas, EPS Secretariat

O.A. Tumanov, Inst. of Spectroscopy, Moscow

L. Van Gerven, University, Leuven

D. Wegener, University, Dortmund

N. Zovko, Rudjer Boskovic Inst., Zagreb

Europhysics Study Conf. Sub-committe

F.A. Gianturco, Inst. of Chemical Physics.

Rome

F. Mueller, Nat. Lab., Los Alamos

Radvanyi, Saturne National Laboratory Gif-sur-Yvette

Chair. A. Messiah

Institut de Recherche Fondamentale

CEN Saclay

F-91 191 Gif-sur-Yvette

T. (6) 9087211

L. Cohen, Inst. of Physics, London

J. Kaczer, Czechoslovak Academy of Sciences, Prague

D. Kuhn, Institute of Experimental

Physics, Innsbruck

G. Landwehr, University, Würzburg

E.W.A. Lingeman, NIKHEF, Amsterdam

C. Rizzuto, University, Genova

G. Thomas, EPS Secretariat

\section{Physics and Society}

Chair. D.H. Parkinson

South Bank

47 Abbey Road

Malvern, WR14 3HH, UK

T. (6845) 5423

Sec. K. Schultze

Physikalisches Institut III

RWTH Aachen

Physikzentrum, Sommerfeldstrasse

D-5100 Aachen

T. (241) 807330

G.J. Béné, University, Geneva

I. Brandus, Central Institute of Physics,

Bucharest

K.H. Chang, University, Amsterdam

G.J. Csikai, University, Debrecen

B. Escoubes, CRN, Strasbourg

R. Habel, ENEA, Frascati

M.J. Higatsberger, University, Vienna

D. Horn, University, Tel-Aviv

P. Jakobsson, Reykjavik

J. Konopka, University, Warsaw

G. Landwehr, University, Würzburg

E. Lillethun, University, Bergen

E.W.A. Lingeman, NIKHEF, Amsterdam

O. Piller, Fed. Office for Metrology, Bern

I. Slaus, Rudjer Boskovic Inst., Zagreb

G.A. Smolensky, A.F. loffe Physico-

Technical Institute, Leningrad

L. Stoll, Technical Institute, Prague

A. Tartaglia, Dept. of Physics, Turin

M. Tomak, M.E.T. Univ. Ankara

W. Turkenburg, University, Utrecht

\section{Europhysics News}

Chair. J. Muller

Section de Physique

Université de Genève,

Quai Ernest-Ansermet 24

$\mathrm{CH}-1211$ Geneva 4

T. (22) 219355

Sec. E.N. Shaw

EPS, POB 69

$\mathrm{CH}-1213$ Petit-Lancy 2

T. (22) 931130

K. Appert, Plasma Physics Research Centre, Lausanne

J. Auth, DDR National Committee

for Physics, Berlin

- A. Baratoff, IBM Res. Lab., Rüschlikon

G.J. Béné, University, Geneva

A.B. Berezin, A.F. loffe Physico-

Technical Institute, Leningrad

E. Burzo, Central Institute of Physics, Bucharest

J. Campos, University, Madrid

J. Charap, Queen Mary College, London

F. De Martini, University, Rome

E. Dreisigacker, Physik-Verlag

Weinheim

M. Erbudak, ETH, Zürich

A. Gotsman, University, Tel-Aviv
J. Hendekovic, Ruder Boskovic Inst., Zagreb

-B. Jacrot, EMBL, Grenoble

F.J. Kedves, Kossuth University,

Debrecen

- G.R. Macleod, CERN, Geneva

A. Maeder, Geneva Observatory,

Versoix

- M. Mayor, Geneva Observatory, Versoix

W.S. Newman, Geneva

J. Niederle, Czechoslovak Academy of

Sciences, Prague

K. Paulus, Inst. of Physics, Bristol

J. Sharpey-Schafer, University,

Liverpool

T.I. Sigfusson, Univ. of Iceland, Reykjavik

A. Sobiczewski, University, Warsaw

M. Taieb, Palais de la Découverte, Paris

A. Taroni, Department of Physics,

Modena

G. Thomas, EPS Secretariat

F. Troyon, Plasma Physics Research

Centre, Lausanne

$H$. van Krugten, University, Delft

- $=$ Member of Editorial Board

Sec. E.N. Shaw

EPS, POB 69

$\mathrm{CH}-1213$ Petit-Lancy 2

T. (22) 931130

G. Alaga, University, Zagreb

K.F. Alexander, Institute of Electron

Physics, Berlin

K. Appert, Plasma Physics Research Centre, Lausanne

D. Barb, Central Institute of Physics, Bucharest

G. Bellini, Institute of Physical Sciences, Milan

A.B. Berezin, A.F. loffe PhysicoTechnical Institute, Leningrad

P.S.H. Bolman, North-Holland Publ. Co. Amsterdam

J. Campos, University, Madrid

B.R. Coles, Imperial College of Science and Technology, London

H. Demiryont, Faculty of Physics, Istanbul

J. Devreese, University, Antwerp

C. Flytzanis, Ecole Polytechnique, Palaiseau

A. Gibson, JET, Abingdon

A. Gotsman, University, Tel-Aviv

H.K. Gudmundsson, Science Institute, Reykjavik

J. Kaluzny, Pedagogical Faculty, Trnava

N. Kurti, Europhys. Lett., Oxford

H. Lotsch, Springer-Verlag, Heidelberg 


\section{EPS Divisions}

\section{Astronomy and Astrophysics Division (359)}

Chair. E. Preuss

Max-Planck-Institut

für Radioastronomie

Auf dem Hügel 69

D-5300 Bonn 1

T. (228) 525280 TX. 886440 mpifr d

Sec. J.-P. Swings

Institut d'Astrophysique

Université de Liège

B-4200 Cointe-Lièg

T. (41) 529980 Tx. 41264 astrlg b

\section{Board Members}

J. Kota, Central Research Institute for Physics, Budapest

N. Lund, Danish Space Research

Institute, Lyngby

H. Ögelman, Middle East Technical University, Ankara

K. Pounds, University, Leiceste

M. Tarenghi, ESO, Garching

J. Trümper, Max-Planck Institute for Extraterrestrial Physics, Garching

J. van Paradijs, Astronomical Institute,

Amsterdam

G.T. Zatsepin, Lebedev Institute, Moscow
C. Cesarsky, CEN-Saclay, Gif-sur-Yvette

\section{Solar Physics Section (122)}

Chair. N. Weiss

Department of Applied Mathematics and Theoretical Physics

University, Silver Street

Cambridge CB3 9EW, England T. (223) 51645

Sec. A. Benz

Institut für Astronomie

ETH

$\mathrm{CH}-8092$ Zürich

T. (1) 2564223

Board Members :

F. Chiuderi-Drago, Observatory, Meudon

J. Christensen-Dalsgaard, Nordita, Copenhagen

F.L. Deubner, University, Würzburg

W. Mattig, Kiepenheuer Institute

Freiburg

R.W.P. McWhirter, Rutherford Appleton Laboratory, Chilton

M.A. Pick, Observatory, Meudon E.R. Priest, University, St Andrews B. Schmieder, Observatory, Meudon

E.H. Schröter, Kiepenheuer Institute, E.H. Schro
Freiburg

\section{Nuclear Physics Division (589)}

Chair. G. Goldring

Weizmann Institute

Rehovot 76100 , Israel

T. (8) 482528

Sec. R.M. Bock

G.S.I. - Ges. für Schwerionenforschung $\mathrm{mbH}$

Postfach 110541

D-6100 Darmstadt 11

T. (6151) 359760

Vice-Chair.: G. Tibell

Gustaf Werners Institute, Uppsala

\section{Board Members:}

R. Bergere, CEA, Saclay

A. Berinde, Central Institute of Physics, Bucharest

P. Glaudermans, University, Utrecht

I. Iori, Institute of Physics, Milan

J. Kantele, University, Gyväskylä

P. Lieb, University, Göttingen

G. Morrison, University, Birmingham

T. Paradellis, University, Athens

I. Sick, University, Basel

1. Ulehla, Charles Univ., Prague

P. von Brentano, University, Cologne

H. Zingl, University, Graz
Computational Physics Group (386)

Chair. R.S. Peckover

Safety and Reliability Directorate UKAEA, Wigsham Lane, Culcheth Warrington WA $34 \mathrm{NE}$, England T. (925) 31244 ext. 7338

Sec. P. Van Binst

Université Libre de Bruxelles

Bd. du Triomphe, POB 230

B-1050 Bruxelles

T. (2) 6413211

Board Members :

R.K. Bock, CERN, Geneva

R. Gruber, EPFL, Lausanne

J. Linderberg, Dept. of Chemistry

University, Aarhus

J. Nadrchal, Institute of Physics,

CVAS, Prague

J. Nuhrenberg, MPI for Plasma Physics, Garching

P. Pyykko, University, Helsinki

A.G. Tenner, NIKHEF-H, Amsterdam

B.P. Veltman, University of Technology, Delft

High Energy and Particle Physics Division (548)

Chair. R.A. Salmeron

ab. de Physique Nucléaire

des Hautes Energies

Ecole Polytechnique

F-91128 Palaiseau Cedex

T. (6) 9418200

Sec. W. Barte

DESY

Notkestrasse 85

- 2000 Hamburg 52

T. (40) 89980

Board Members :

G. Altarelli, University La Sapienza, Rome

G. Bellini, University, Milan

H. Bøggild, Niels Bohr Institute Copenhagen

R. Cashmore, University, Oxford

J.M. Charap, Queen Mary College, London

Chair. S. Leach

Labor. de Photophysique Moléculaire

Bâtiment 213, Université de Paris-Sud

F-91 405 Orsay Cedex

Institut für Angewandte Physik

Universität Bonn

Wegelerstrasse 8

D-5300 Bonn 1

T. (228) $733477 / 8$

Mruislaan 407

NL-1098 SJ Amsterdam

T. (20) 946711

\section{Board Members:}

U.V. Afrosimov, USSR Academy of

Sciences, Leningrad

N. Andersen, University, Copenhagen

M. Barat, Orsay, Paris

J. Connerade, Imperial College, London

G. Dohnalik, University, Cracow

H. Erhardt, University, Kaiserslautern

I. Hertel, University, Berlin West

D.N. Stacey, University, Oxford

F. Wuilleumier, University Paris-Sud, Orsay

The Chairmen and Secretaries of the Sections serve also on the Divisional Board.

\section{Chemical Physical Section (283)}

Chair. E.A.C. Lucken

Département de Chimie Physique

Sciences II, 30 quai E.-Ansermet

$\mathrm{CH}-1211$ Geneva 4

T. (22) 219355

\section{Board Members :}

R. Daudel, Applied Wave

Mechanics Centre, Paris

R. Gaspar, Kossuth Univ., Debrecen

L. Jansen, Battelle Institute, Geneva

E. Roth, CEN-Saclay, Gif-sur-Yvette

J.P. Toennies, MPI for Fluid Mechanics,

Göttingen

A. van der Avoird, University, Nijmegen

G. Wagnière, University, Zurich

D.A. Young, Chemical Soc., London

\section{Sec. F.G. Meije}

Zeeman Laboratorium

Universiteit van Amsterdam

Plantage Muidergracht 4

NL-1018 TV Amsterdam

$T$ (20) 5222161

\section{Board Members:}

E. Arimondo, University, Pisa

E. Bernabeu, University, Madrid

J. Desesquelles, University, Lyon

S. Legowski, University, Torun

J.D. Silver, Clarendon Lab., Oxford

S. Svanberg, Institute of Technology,

Lund

R.C. Thompson, National Physical Lab.,

London

G. von Oppen, Technical University,

Berlin West

J.F. Wyart, Aimé Cotton Lab., Orsay

T. (6) 9417909

Sec. S. Stolte

Fysisch Laboratorium

Katholieke Universiteit

Toernooiveld

NL-6525 ED Nijmegen

T. (80) 558833

Board Members:

S. Califano, University, Florence

R.N. Dixon, University, Bristol

P. Erman, Royal Institute of Technology Stockholm

B. Jezowska, University, Wroclaw

1. Kovacs, Polytechnic, Budapest

V.S. Letokhov, Academy of Sciences, Moscow

I.M. Mills, University, Reading

Z. Pajak, University, Poznan

S.D. Peyerimhoff, University, Bonn

M. Winnewisser, University, Giessen

H.C. Wolf, University, Stuttgart

B. Zuev, Academy of Sciences, Tomsk

Electronic and Atomic Collisions Section (361)
Chair. C.J. Joachain

Faculté des Sciences

Université Libre de Bruxelles

227 Boulevard du Triomphe

B-1050 Bruxelles

T. (2) 6490030

Sec. A. Giardini-Guidoni

ENEA, Tib-Fis, Laboratorio Spettro-

scopia Molecolare, POB 65 .

1-000 44 Frascati

T. (6) 942104
Board Members :

J.-P. Gauyacq, Univ. Paris-Sud, Orsay M. Kurepa, Inst. of Physics, Belgrade F. Linder, University, Kaiserslautern W. Lindinger, University, Innsbruck V. Martisovits, University, Bratislava M.R.C. McDowell, Royal Holloway

College, Egham

G. Nienhuis, RU, Utrecht

K. Taulbjerg, University, Aarhus

L. Wolniewicz, University, Torun
T. Ekelof, University Uppsala

J. Fischer, CSAV, Prague

M. Gourdin, Pierre et Marie Curie

University, Paris

A. Martin, CERN, Geneva

J.C. Sens, NIKHEF, Amsterdam

R. Sosnowski, Inst. of Nuclear

Research, Warsaw

D. Wegener, University, Dortmund

F.J. Yndurain, Autónoma University, Madrid

\section{Optics Division (55)}

Chair. H. Tizian

Institut für Technische Optik

Universität Stuttgart

Pfaffenwaldring 9

D-7000 Stuttgart 80

T. (711) 6856075

Sec. H.A. Ferwerda

Department of Applied Optics

University of Groningen

Nijenborg 18

NL-9747 AG Groningen

T. (50) 115959

\section{Provisional Board:}

J. Bescos, Optics Institute, Madrid

K. Biedermann, Royal Institute of

Technology, Stockholm

J. Bulabois, Franche-Comté University,

Besançon

J.P. Huignard, Thomson-CSF, Orsay

E.R. Pike, Royal Signal \& Radar

Establishment, Malvern

W. Schmidt, Carl Zeiss, Oberkochen 
Chair. J. Devreese

Department of Physics

Universiteit Antwerpen (UIA)

Universiteitsplein 1

B-2610 (Wilrijk) Antwerpen

T. (3) 8282528

Sec. V. Van Doren

Universiteit Antwerpen (RUCA

17 Groenenborgerlaan

B-2020 Antwerpen

T. (3) 2180317

Board Members:

A. Abrikosov, USSR Academy of

Sciences, Moscow

S. Bratos, Pierre et Marie Curie Univ., Paris

R. Cahn, Cambridge

H. Grimmeiss, Inst. of Technology, Lund

G. Harbeke, RCA, Zurich

V. Heine, Cavendish Lab., Cambridge

G.M. Kalvius, Technical Univ. Munich

A. Keller, University, Bristol

S.O. Lundqvist, Institute of Theoretical

Physics, Göteborg

E. Mooser, EPFL, Lausanne

F.M. Mueller, Nat. Lab., Los Alamos,

USA

D. Thoulouze, CRTBT - CNRS, Grenoble

R. Ross, E. Anglia University, Norwich

M. Weger, Hebrew University,

Jerusalem

R.F. Willis, Cavendish Lab., Cambridge

G. Winstel, Siemens AG, Munich

A. Zawadowski, Central Institute

for Physics, Budapest

\section{Liquids Section (47)}

Chair. S. Bratos

Université Pierre et Marie Curie

Tour 16, 4 place Jussieu

F-75230 Paris Cedex 05

T. (1) 3295574

Sec. J-CI. Leicknam

Université Pierre et Marie Curie

Tour 16, 4 place Jussieu

F-75230 Paris Cedex 05

T. (1) 3295574

Board Members :

F. Barocchi, Department of Physics, Florence

Th. Dorfmüller, University, Bielefeld

E. Guyon, Ecole Supérieure of Physics

and Chemistry, Paris

W. Helfrich, Free University, Berlin West

J. Janik, Institute of Nuclear Physics, Cracow

P. Mazur, Lorentz Institute, Leiden

G. Nicolis, Free University, Brussel

J.S. Rowlinson, University, Oxford

M.G. Velarde, University of Education

at a Distance, Madrid

Ya. Zeldovitch, Space Research

Institute, Moscow

Low Temperature Section (377)

Chair. D. Thoulouze

Centre de Recherches sur les Très

Basses Températures - CNRS

25 ave. des Martyrs, $166 \times$

F-38042 Grenoble Cedex

T. (76) 969837 ext. 300

Committee Members:

A.S. Borovik-Romanov, Inst. for Physical Problems, Moscow

Y. Bruynseraede, Catholic University, Leuven

I. Kirschner, Eötvös Roland University,

Budapest

M. Krusius, University, Turku

H.R. Ott, ETH, Zürich

F. Pobell, KFA, Jülich

H. Postma, University, Delft

F.B. Rasmussen, H.C. Orsted Inst.

Copenhagen

G.L. Romani, Inst. of Solid State

Electronics, Rome

W.F. Vinen, University, Birmingham
Macromolecular Physics Section (222)

Chair. A. Keller

H.H. Wills Physics Laboratory

University of Bristol

Royal Fort, Tyndall Ave.

Bristol BS8 1TL, England

T. (272) 24161

Committee Members:

F.J. Baltà-Calleja, Institute of Chemical

Physics, Madrid

G. Bodor, Polymer Research Institute

Budapest

L.L. Chaboy, Tech. Univ., Lyngby

H.G. Kilian, University, Ulm

R. Koningsveld, DSM, Geleen

M. Gryszewski, Macromolecular

Studies Centre, Lotz

E. Martuscelli, CNR Polymer Materials,

Naples

J.P. Mercier, High Polymer Lab.

Louvain-la-Neuve

L. Monnerie, ESPCI, Paris

J. Spevacet, Inst. for Macromolecular

Chemistry, Prague

\section{Magnetism Section (487)}

Chair. G.M. Kalvius

Technischen Universität München

Physik E15, James Franck Strasse

D-8046 Garching

T. (89) 3209250

\section{Committee Members:}

L.J. de Jongh, Kammerlingh Onnes

Laboratory, Leiden

R. Elliott, University, Oxford

J. Kaczer, Czechoslovak Acad. of

Sciences, Prague

E. Karlsson, University, Uppsala

S. Methfessel, Ruhr Univ., Bochum

\section{Metals Section (559)}

Chair. R. Cahn

6 Storey's Way

Cambridge CB3 ODT, England

T. (223) 60143

Committee Members :

D. Bacon, University, Liverpoo

D. Buschow, Philips' Research Labs. Eindhoven

O. Fischer, University, Geneva

J.P. Gaspard, University, Liege

M. Hillert, KTH, Stockholm

E. Lüscher, Technical University, Munich

G. Martin, CNRS, Vitry

P. Fulde, MPI for Solid State Physics, Stuttgart

Semiconductors and Insulators (654)

Chair. G. Harbeke

RCA Laboratories Ltd

Badenerstrasse 569

$\mathrm{CH}-8048$ Zürich

T. (1) 526350

Committee Members:

J.W. Allen, University, St. Andrews

D. Bäuerle, University, Linz

F. Beleznay, Institute for Technical

Physics, Budapest

R. Blinc, Josef Stefan Inst., Ljubljana

A. Frova, University, Rome

H. Grimmeiss, University, Lund

P. Günter, ETH, Zürich

D. Kaplan, Thomson SA, Orsay

S. Porowski, Academy of Sciences, Warsaw

\section{Surfaces and Interfaces (456)}

Chair. R.F. Willis

Cavendish Laboratory

Madingley Road

Cambridge, $\mathrm{CB} 3 \mathrm{OHE}$, England

T. (223) 66477

Committee Members:

G. Benedek, Inst. of Physics, Milan

V. Bortolani, University, Modena

A.M. Bradshaw, Fritz-Haber Institute Berlin West

N. Garcia, Automa University, Madrid

K. Horn, Fritz-Haber Institute,

Berlin West

F. Koch, Tech. University, Munich

M. Landolt, ETH, Zurich

B.I. Lundqvist, Chalmers Univ. Göteborg

C. Sébenne, Pierre et Marie Curie University, Paris

J.F. van der Veen, FOM, Amsterdam

K. Wojciekowski, University, Wroclaw

Plasma Physics Division (335)

Chair. A. Gibson

JET Joint Undertaking

Abingdon, Oxford OX14 3EA, England

T. (235) 28822

Vice-Chair. D. Sweetman

Culham Laboratory

Abingdon, Oxford OX14 3DB, England

Treas. K. Appert

Centre de Recherches en Physique

des Plasmas, 21 Ave. des Bains

$\mathrm{CH}-1007$ Lausanne

T. (21) 473453

University of Berne

Sidlerstrasse 5
Quantum Electronics Division (305)

Chair. C.A. Sacchi

Istituto di Fisica del Politecnico

Piazza Leonardo da Vinci 32

1.20100 Milano

T. (2) 295611 Tx. 333467 polimi i

Sec. H.P. Weber

Institute of Applied Physics

$\mathrm{CH}-3012$ Bern

T. (31) 658931 Tx. 32320 phybe

Board Members :

F.R. Aussenegg, University, Graz

M.J. Colles, Heriot-Watt University, Edinburgh

F. de Martini, G. Marconi Institute,

Rome

- A. Dönszelmann, Zeeman Laboratory, Amsterdam

C. Flytzanis, Ecole Polytechnique,

Palaiseau

J. Jerphagnon, CNET, Lannion

V.S. Letokhov, Institute of

Spectroscopy, Moscow

R. Loudon, Essex University, Colchester

M. Sigrist, ETH, Zürich

S. Stenholm, University, Helsinki

-S. Svanberg, Institute of Technology, Lund

H. Walther, University, Garching

$H$. Welling, Technical University,

Hannover

- B. Wilhelmi, University, Jena

- Coopted members

Board Members:

R. Behrisch, MPI for Plasma Physics,

Garching

W. Boetticher, University, Hannover

$\mathrm{H}$. de Kluiver, FOM, Nieuwegein

F. Engelmann, FOM, Nieuwegein

J. Jacquinot, CEN, Fontenay-aux-Roses

V. Jensen, Risø National Lab., Roskilde

M. Kaufmann, MPI for Plasma Physics, Garching

R.V. Sagdeev, USSR Academy of

Sciences, Moscow

D.C. Schram, University of Technology, Eindhoven

\section{Associate Members}

Agfa-Gevaert N.V., Mortsel, Belgium

LAir Liquide, Jouy en Josas, France

Battelle Geneva Research Centre,

Carouge-Geneva, Switzerland

BESSY - Berliner Elektronenspeicherring Ges.

für Synchrotronstrahlung, Berlin West

British Telecom Research Laboratories,

Martlesham Heath, England

CEA - Commissariat à l'Energie Atomique,

Paris, France

CEGB - Central Electricity Generating Board,

London, England

Cerberus Ltd., Männedorf, Switzerland

CERN - European Organization for Nuclear

Research, Geneva, Switzerland

CGE - Cie Générale d'Electricité,

Paris, France

CISE - Centre for Information, Studies and Experiment, Milan, Italy

CNES - Centre National d'Etudes Spatiales, Paris, France

CNET - Centre National d'Etudes des Télécommunications, Lannion, France

CNR - Consiglio Nazionale delle Ricerche, Rome, Italy 
CNRS - Centre National de la Recherche Scientifique, Paris, France

DESY - Deutsches Elektronen-Synchrotron, Hamburg, FRG

ECN - Netherlands Energy Research Foundation, Petten, the Netherlands

EDF - Electricité de France, Paris, France

ELF - Société Nationale ELF Aquitaine, Paris, France

ELSAG - Elettronica San Giorgio, Genoa, Italy

ENEA - Comitato Nazionale per la Ricerca e Sviluppo dell'Energia Nucleare e delle Energie Alternative, Rome, Italy

ESA - European Space Agency, Paris, France

$\mathrm{FIZ}$ Energie, Physik, Mathematik GmbH, Eggenstein-Leopoldshafen, FRG

FOA - National Defence Research Institute, Stockholm, Sweden

FOM - Foundation for Fundamental Research on Matter, Utrecht, NL

General Electric Co. Hirst Research Centre, Wembley, England

GSI - Gesellschaft für Schwerionenforschung, Darmstadt, Fed. Rep. of Germany

Hahn-Meitner-Institut für Kernforschung, Berlin-West

Haldor Topsøe, Lingby, Denmark

IBM Europe, Paris, France

ILL - Institut Max von Laue-Paul Langevin, Grenoble, France

INFN - Istituto Nazionale di Fisica Nucleare, Rome, Italy

Institute for Energy Technology Kjeller, Norway

JET Joint Undertaking, Abingdon, England

N.V. Kema, Arnhem, the Netherlands

Kernforschungszentrum Karlsruhe GmbH, Karlsruhe, Fed. Rep. of Germany

KFA - Kernforschungsanlage Jülich, Jülich, Fed. Rep. of Germany

KFKI-Central Institute for Physics, Budapest, Hungary

Masson Editeur, Paris, France

Max-Planck-Institut für Festkörperforschung. Stuttgart, Fed. Rep. of Germany

Delegates to Council:
Max-Planck-Institut für Plasmaphysik, Garching, Fed. Rep. of Germany

MOM - Hungarian Optical Works, Budapest, Hungary

NORDITA - Nordisk Institut for Teoretisk Atomfysik, Copenhagen, Denmark

North-Holland Publishing Co.,

Amsterdam, the Netherlands

Officine Galileo, Florence, Italy

Oldelft, Delft, the Netherlands

Pechiney-Ugine-Kuhlmann, Paris, France

Compagnie Française Philips,

Limeil-Brévannes, France

N.V. Philips' Gloeilampenfabrieken, Eindhoven, the Netherlands

Physikalisch-Technische Bundesanstalt, Braunschweig, Fed. Rep. of Germany

Plessey Research (Caswell) Ltd.,

Towcester, England

Laboratories RCA Ltd., Zürich, Switzerland

Rhône-Poulenc, Courbevoie, France

Risø National Laboratory, Roskilde, Denmark

Cie. de Saint-Gobain,

Pont-à-Mousson, France

Siemens AG, Berlin \& Munich, FRG

SIN - Swiss Institute for Nuclear Research, Villigen, Switzerland

Springer-Verlag GmbH \& Co. KG, Heidelberg, Fed. Rep. of Germany

Standard Telecommunication Laboratories, Harlow, England

Taylor \& Francis Ltd., London, England

Thomson SA, Paris, France

TNO - Netherlands' Organization for Applied Scientific Research, The Hague, NL

TUNGSRAM - United Incandescent Lamp and Electrical Company Limited, Budapest, Hungary

UK Atomic Energy Authority London, England

URANIT GmbH

Jülich, Fed. Rep. of Germany

Vacuumschmelze $\mathrm{GmbH}$,

Hanau, Fed. Rep. of Germany

R.W. Brander, British Telecom, Ipswich

E. Feldtkeller, Siemens, Munich

J.A. Goedkoop, ECN, Petten

J.C. Lehmann, CNRS, Paris

\section{Meetings Up-date}

\section{5}

\section{4-6 Nov.}

Grenoble, France

International Workshop on Evaluation of

Single-Crystal Diffraction Data from

2-D Position Sensitive Detectors

Mrs. R. Mason, Inst. Laue-Langevin,

156 X, F-38042 Grenoble Cedex

T. (76) 487226 , Tx. ILL $320621 \mathrm{~F}$

50 / FF 300.-

\section{2-4 Dec.}

Tucson, AZ, USA

Topical Meeting on Optical Bistability (OB3)

Optical Society of America, Meetings Dept.,

1816 Jefferson Place, N.W., Washington, DC 20036

Ab: 1.8.85/PP / US \$ 170. - before 1.11.85; 190. - after; US $\$ 70 .-$ students, before $1.11 .85 ; 90 .-$ after

\section{6}

1-5 July

Heidelberg, FRG

Int. Symp. on Weak and Electromagnetic Interactions in Nuclei

H.V. Klapdor, Max-Planck-Inst. f. Kernphysik, POB 103980, D-6900 Heidelberg

\section{7}

31 Aug. - 4 Sept. Brussels, Belgium 6th Int. Symp. on Capture Gamma-Ray

Spectroscopy and Related Topics

P. Van Assche, Nuclear Energy Centre,

SCK/CEN, B-2400 Mol

$\mathrm{PP} / 250$

\section{DECEMBER 2-4, 1985}

The topical meeting on optical bistability is intended to provide an international interdisciplinary forum for the exchange of knowledge on the progress of various aspects of optical bistability and optical nonlinearities. The conference sessions will include optical bistability, multistabilities and instabilities. The three day program will consist of a combination of lectures (invited and contributed) and poster sessions.

Papers in the following areas are solicited:

- Fundamental properties of systems having strong light-matter

interactions

- Instabilities and optical chaos

- Transverse and longitudinal effects: diffraction, absorption, diffusion, conduction, etc.

- Physical mechanisms and experimental determinations of large optical nonlinearities

- New schemes and models for optical bistability

- Bistability and instabilities in semiconductor lasers

- Operating characteristics and fundamental limits of nonlinear optical logic devices: etalons, waveguides, etc.

- Parallel nonlinear processing optical data

- Optical computing: interconnects, input, clocking, and output - with an emphasis on nonlinear devices

For more information contact: Optical Society of America 1816 Jefferson Place, N.W. Washington, D.C. 20036 202/223-8130

OSA Meetings Department 202/223-0920

Abstract Deadline: August 1, 1985 